
all forms.

\title{
The Multibeam Radar Sensor Birales: Performance Assessment for SPACE Surveillance AND Tracking
}

\author{
Matteo Losacco ${ }^{a *}$, Pierluigi Di Lizia ${ }^{a}$, Mauro Massari ${ }^{a}$, \\ Andrea Mattana ${ }^{\mathrm{b}}$, Federico Perini ${ }^{\mathrm{b}}$, Marco Schiaffino ${ }^{\mathrm{b}}$, Claudio Bortolotti ${ }^{\mathrm{b}}$, Mauro \\ Roma $^{\mathrm{b}}$, Giovanni Naldi ${ }^{\mathrm{b}}$, Giuseppe Pupillo ${ }^{\mathrm{b}}$, Germano Bianchi ${ }^{\mathrm{b}}$, Luca Lama $^{\mathrm{b}}$, \\ Denis Cutajar ${ }^{\mathrm{c}}$, Alessio Magro ${ }^{\mathrm{C}}$, \\ Claudio Portelli ${ }^{\mathrm{d}}$, \\ Marco Reali ${ }^{\mathrm{e}}$, Walter Villadei ${ }^{\mathrm{e}}$
}

a Department of Aerospace Science and Technology, Politecnico di Milano, Via G. La Masa 34, 20156 Milano, Italy, \{matteo.losacco, pierluigi.dilizia,mauro.massari\}@polimi.it

b Italian National Institute for Astrophysics - Institute for Radio astronomy, Via P. Gobetti 101, 40129 Bologna, Italy, \{a.mattana, f.perini, m.schiaffino, c.bortolotti, m.roma, g.naldi, g.pupillo, g.bianchi\}@ira.inaf.it

c University of Malta, Msida MSD 2080, Malta, \{denis.cutajar, alessio.magro\}@um.edu.mt

d Italian Space Agency, Via del Politecnico snc, 00133 Roma, Italy, claudio.portelli@asi.it

e Italian Air Staff - ITAF, Viale dell'Università 4, 00185 Roma, Italy, \{marco.reali@aeronautica, walter.villadei@am\}.difesa.it

* Corresponding Author

\begin{abstract}
Near-Earth space has become progressively more crowded in active satellites, inactive spacecraft and debris. Consequently, an international effort is currently being devoted to improving the performance of the network of optical and radar sensors for space objects monitoring. Within this framework, the use of the novel bistatic radar sensor BIRALES is investigated in this work. The tailored orbit determination algorithm is described, with a detailed description of the transiting object trace definition method. The second part of the work illustrates the results achieved with both numerical simulations and observation campaigns. The sensor performance is assessed considering both cases of known and unknown objects. For all cases, the effect of measurement noise on each single available measurement is investigated. Finally, the results achieved during satellite Tiangong-1 re-entry observation campaign are shown.
\end{abstract}

Keywords: Space debris, orbit determination, radar sensors.

\section{Acronyms/Abbreviations}

- Doppler shift (DS)

- Fast Fourier Transform (FFT)

- Field of view (FoV)

- Field Programmable Gate Array (FPGA)

- Orbit determination (OD)

- Radio Frequency Transmitter (RFT)

- Receiver (RX)

- Signal-to-Noise Ratio (SNR)

- Slant range (SR)

- Space Situational Awareness (SSA)

- Space Surveillance and Tracking (SST)

- Technical Data Management (TDM)

- Transmitter (TX)

- Two-Line Elements (TLE)

\section{Introduction}

The number of manmade objects orbiting the Earth has dramatically increased during the last decades, posing a serious risk for space-based activities. Most of these objects are classified as space debris and include inactive satellites, discarded launch stages, and fragments originated from satellite breakups and collisions. Several counter measures have been adopted with the aim of reducing mission related risks and to control the number of objects in orbit. In parallel, specific space programs were started to build the expertise required to manage the challenges posed by the space traffic control problem. Collision risk assessment is performed daily by satellite operators who are provided with conjunction summary messages to support decisions on the execution of collision avoidance manoeuvres 1. In addition, re-entry predictions of objects are regularly produced to estimate on ground risks 2]. Both collision risk assessment and re-entry predictions rely on the accurate estimation and prediction of the state of the orbiting objects, which are derived from the tracking of 
69th International Astronautical Congress (IAC), Bremen, Germany, 1-5 October 2018.

Copyright (C) 2018 by Politecnico di Milano. Published by the IAF, with permission and released to the IAF to publish in all forms.

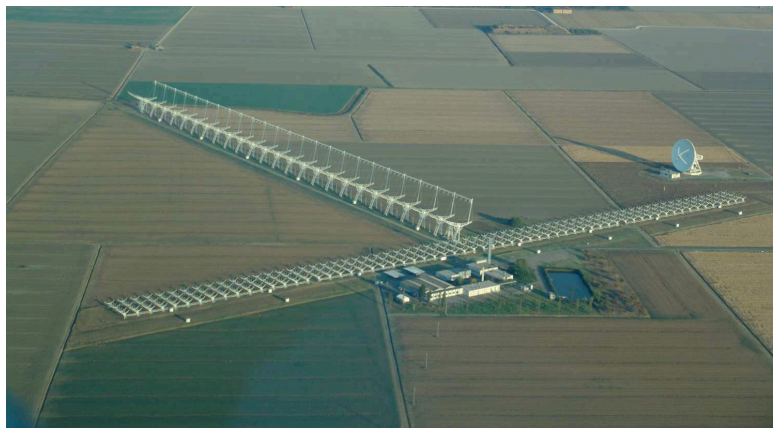

Fig. 1: A view of the Medicina Radio astronomical Station. In the foreground, the Northern Cross.

the space objects using dedicated optical, radar and laser sensors.

Survey and tracking of objects in Earth orbit is one area where the European Space Surveillance and Tracking (SST) Support Framework and the European Space Agency (ESA) Space Situational Awareness (SSA) programmes are active [3.4], and the implementation of a European network of sensors represents one of the main objectives. In this regard, the Italian Northern Cross radio telescope array has been upgraded to serve the European SST Framework in the frame of the BIstatic RAdar for LEo Survey (BIRALES) sensor [5]. BIRALES uses part of the Northern Cross radio telescope located in Medicina (Bologna, Italy) as the receiver (see Fig. 1). Part of the radio telescope has been refurbished and a digital back end has been implemented to allow beamforming of 32 beams distributed across the receiver field of view (FoV). When an object transits inside the antenna FoV, the beams are illuminated by the reflected radio wave. Consequently, besides the classical range and Doppler shift measurements, the beam illumination sequence provides an estimate of the transit direction of the object in terms of angular deviation with respect to the nominal receiver pointing direction. The data received from BIRALES are provided as input to a tailored orbit determination (OD) algorithm, which is aimed at computing an estimate of the orbital parameters of the observed object.

A numerical simulator of BIRALES has been developed to assess its performance through dedicated simulations. Given a catalogue of space objects, the simulator identifies the passages of all the objects in the sensor FoV during a simulated observation campaign. Then, the simulated measurements are generated for each passage and are provided as input to the OD module, along with the required transmitter and receiver information. The simulator is designed so that different kinds of transmitter and beams geometry can be easily defined by the user.

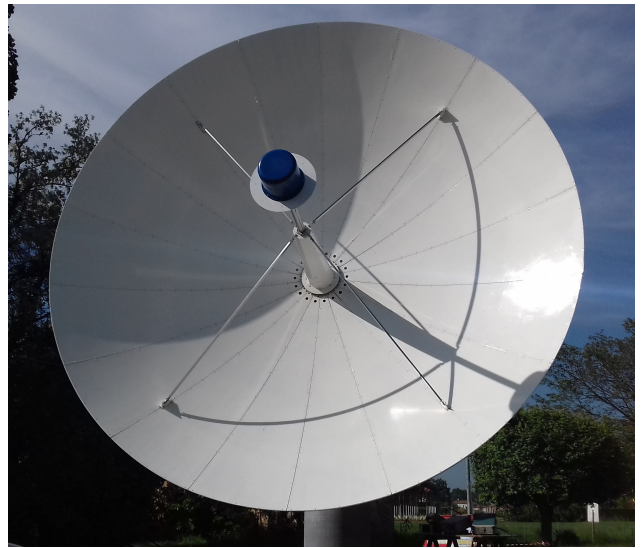

Fig. 2: Radio Frequency Transmitter.

This enables an effective analysis of the sensitivity of the sensor to its configuration.

The first part of this paper is dedicated to the description of BIRALES sensor. Then, a detailed illustration of the OD algorithm is offered, with the trace reconstruction algorithm representing the core of the method. Finally, the performance of the sensor is presented as obtained with both numerical simulations and real observation campaigns.

\section{BIRALES sensor}

BIRALES sensor uses a bistatic configuration. The "Radio Frequency Transmitter" (RFT) located at the Italian Joint Test Range of Salto di Quirra (PISQ) in Sardinia is used as transmitter (Fig. 2), and the Northern Cross radio telescope of the radio astronomy station of Medicina (BO) as receiver.

The RFT consists of a powerful amplifier able to supply a maximum power of $10 \mathrm{~kW}$ in the bandwidth $410-415 \mathrm{MHz}$. It is a $7 \mathrm{~m}$ dish completely steerable at a maximum speed of $3 \mathrm{deg} / \mathrm{s}$ and with right-hand circular polarization. Its $\mathrm{FoV}$ matches almost perfectly the receiving antenna one, with a beam of $6 \mathrm{deg}$. The receiving antenna is a portion of the Northern Cross Radio Telescope (Fig. 3), which is currently one of the largest UHF-capable

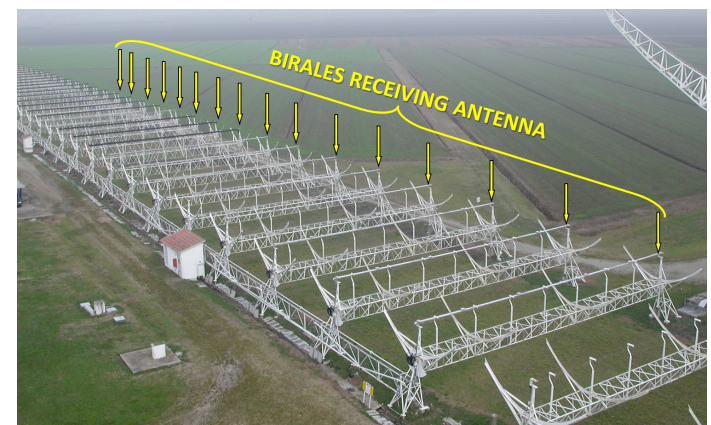

Fig. 3: BIRALES receiving antenna. 


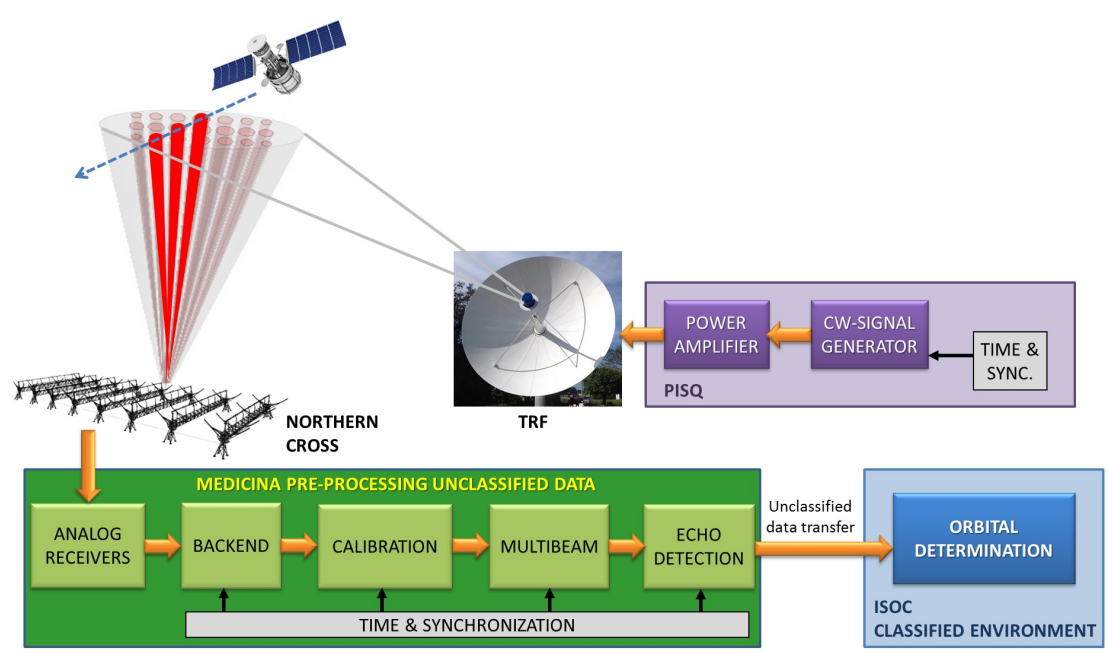

Fig. 4: BIRALES system architecture

antennas in the world, being located at the Medicina Radio Astronomical Station, near Bologna, in Northern Italy 6]. It is owned by the University of Bologna but managed and operated by the Institute for Radio astronomy at the Italian National Institute for Astrophysics (INAF-IRA). It consists of two perpendicular branches: the East-West (E/W) arm is $564 \mathrm{~m}$ long and consists of a single cylindrical antenna with a width of $35 \mathrm{~m}$, whereas the NorthSouth $(\mathrm{N} / \mathrm{S})$ branch is made of 64 parallel antennas with a length of $23.5 \mathrm{~m}$ and width of $7.5 \mathrm{~m}$ each.

The portion dedicated to BIRALES receiving antenna is actually composed of 16 parabolic cylindrical antennas of the N/S branch (see Fig. 3). The total collecting area is about $2800 \mathrm{~m}^{2}$ and it allows the detection of small objects with sub-metric Radar Cross Section (RCS) at $1000 \mathrm{~km}$ of altitude. The peculiarity of the receiver is represented by the possibility of detecting meridian passages only, as it can be moved in elevation only.

BIRALES works in survey mode and exploits an innovative concept based on multibeam technique. Due to the large numbers of receivers installed on the Northern Cross (4 receivers in each N/S antenna for a total of 64 receivers), the FoV can be populated with many independent beams. When an object transits inside the antenna FoV, beams are illuminated by the reflected radio waves. Thus, by looking at the beam illumination sequence, it is possible to estimate the ground track of the transiting objects, with a higher level of detail with respect to a single-beam system.

The system architecture is shown in Fig. 4. Using the RFT it is possible to transmit a power CW signal able to illuminate the target in LEO. The echo radio reflected by the orbital object is received through the Northern Cross and the acquired analog signal is sent to the pre-processing room by an optical fiber link. The digitized data are processed by means of a data acquisition system based on FPGA boards and CPUs, which digitally assembles measured radar echoes using an FFT in spatial domain in order to evaluate the signal detected in each beam. Therefore, Doppler shift, illumination time, antenna pointing angles and measured power intensity associated to each beam are available as well and they are sent to the Italian SST Operation Centre (ISOC) to estimate the orbit of the object detected in a classified environment.

The sensor has been recently upgraded in order to provide also range measurements. Radar ranging works by transmitting, along with the carrier wave, a chirp of known period and span. The Doppler shift can be estimated by the carrier reflection, and the knowledge of this correction for the underlying chirp and of the transmission epoch provides a time of flight. The estimated time of flight is however affected by an ambiguity of $\mathrm{N}$ chirp periods.

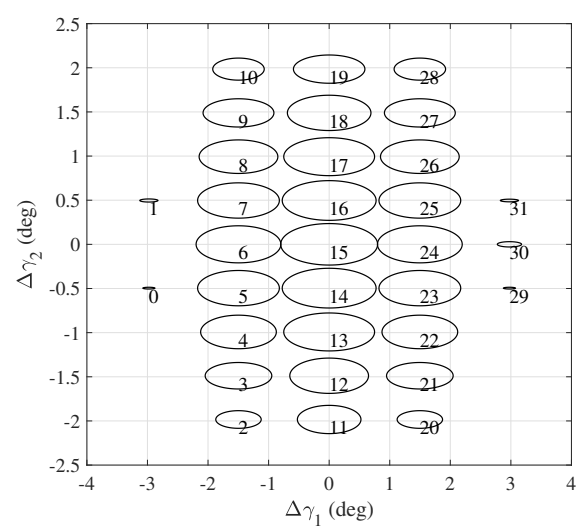

Fig. 5: Medicina receiver beam pattern in terms of angular deviations $\Delta \gamma_{1}$ and $\Delta \gamma_{2}$. 
69th International Astronautical Congress (IAC), Bremen, Germany, 1-5 October 2018.

Copyright (C) 2018 by Politecnico di Milano. Published by the IAF, with permission and released to the IAF to publish in all forms.

This new configuration will be considered for the numerical simulation presented in Section 4.1

\section{Orbit determination}

The starting point for the OD algorithm implemented for BIRALES sensor is represented by a TDM measurement file including, for all the beams illuminated during the object passage, the epoch and the recorded slant range, Doppler shift and Signal-to-Noise Ratio (SNR) measurements. Then, the algorithm is divided in two parts. The first part is dedicated to the estimation of the trace of the object inside the receiver FoV starting from the available SNR and slant range measurements. Then, the state of the object is estimated on the basis of the available slant range and Doppler shift measurements and the estimated trace. In the following paragraphs, both phases are described in detail.

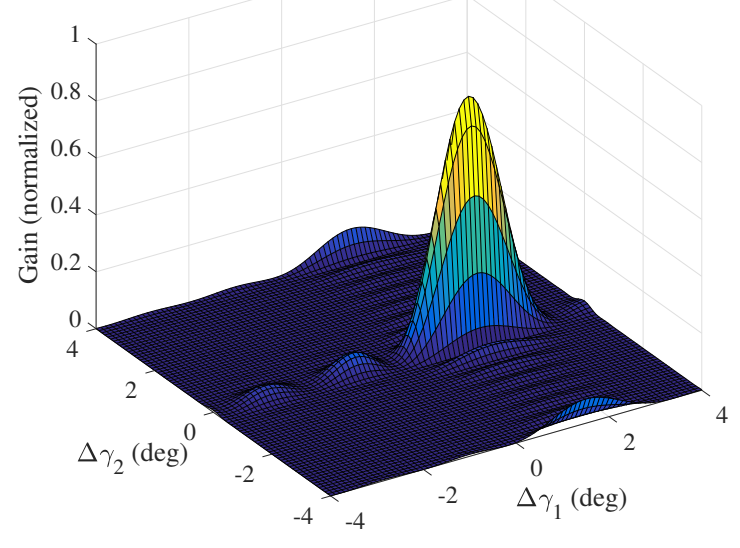

Fig. 6: Gain pattern for beam 24: one main lobe, and other minor side lobes.

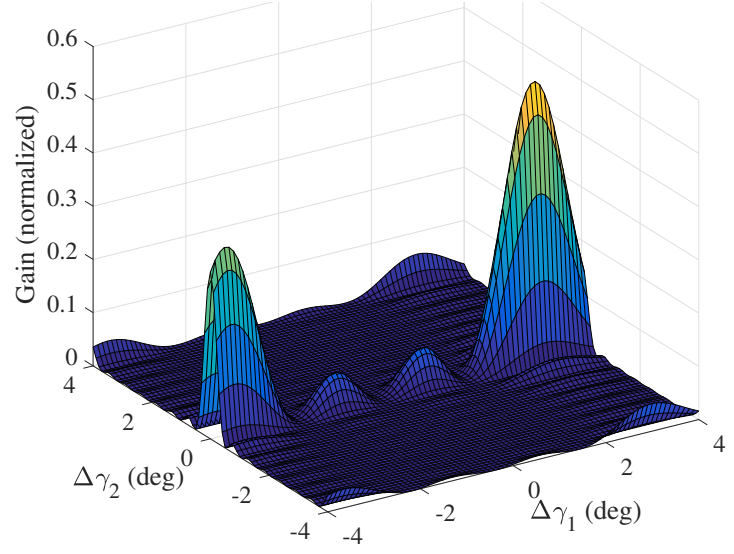

Fig. 7: Gain pattern for beam 30: one main lobe and other not negligible side lobes.

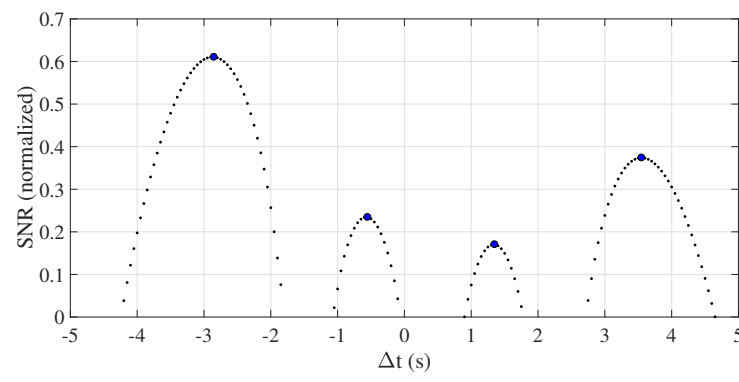

Fig. 8: Beam 30 normalized SNR vs time difference with respect to nominal meridian passage for passage of object NORAD ID 41765, April 20, 2018.

\subsection{Trace definition}

The peculiarity of BIRALES sensor is surely represented by the receiver and its possibility of being populated by 32 beams. Figure 5 shows the distribution of the 32 beams within the receiver FoV. The angles $\Delta \gamma_{1}$ and $\Delta \gamma_{2}$ represent the angular deviations with respect to the receiver nominal pointing. Each single beam typically presents what can be defined a "main lobe", and some other "side lobes" whose entity may be more or less significant according to the investigated beam. The gain pattern of each single beam mainly depends on its position inside the receiver FoV. Beams located close to the line of sight of the sensor typically show side lobes whose size is much lower than the size of the main lobe. An example is given in Fig. 6 for beam 24 , with one main gain peak and other secondary, much smaller, side lobes. These beams will be defined throughout the paper as "dominant beams". On the contrary, beams located at the boundaries of the FoV typically have at least one side lobe whose entity can be compared with the one of the main lobe. Figure 7 shows the gain pattern for beam 30: as can be seen, given the main lobe, there is another gain peak, whose location is more or less symmetric with respect to the $\Delta \gamma_{1}=0$ axis, whose size can be compared with the main lobe, and there are other not negligible secondary peaks.

The peculiarity of the receiver gain pattern has obviously a strong impact on the approach followed in the definition of the object trace inside the receiver FoV. The idea at the basis of the approach is to reconstruct the trace of the object by looking at the sequence of illumination of the beams. This, in turn, essentially consists in matching the measured SNR profile with the one obtained by estimating the trace inside the field of view. The algorithm can be divided in two phases: a first phase S1, in which a first estimate for the object trace is obtained by looking at the recorded SNR peaks, and a second phase $\mathrm{S} 2$, in which the obtained trace is 


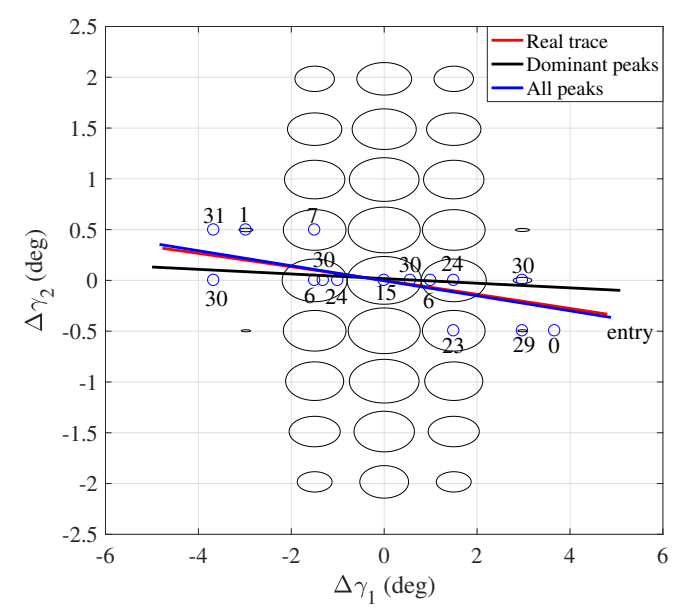

(a)

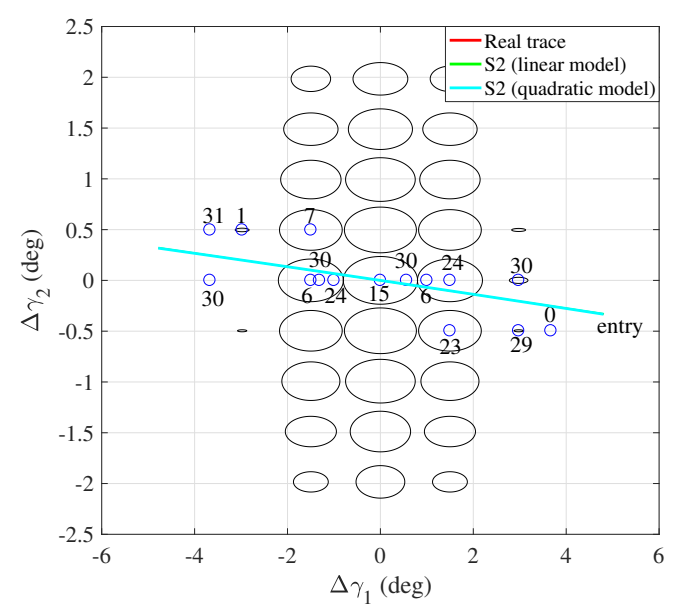

(b)

Fig. 9: Trace definition for object NORAD ID 417654, passage April 20, 2018: (a) step S1, trace definition based on SNR peaks, (b) step S2, nonlinear least-squares on the whole SNR profiles.

used as a first guess for a least-squares fit aimed at minimizing the residuals with respect to the whole SNR profile of all illuminated beams.

The definition of the first guess $\mathrm{S} 1$ is done by looking at all SNR peaks recorded during the passage of the object and associating each signal peak to a given gain peak of a specific beam. This probably represents the most challenging aspect of the trace definition algorithm, given the presence of multiple gain peaks for each beam. When one beam is illuminated, indeed, it is not straightforward to understand which gain peak is responsible for the beam illumination. Figure 8 shows the normalized SNR profile recorded by beam 30 during the passage of object NORAD ID 41765, on April 20, 2018 at 12:41:38.34 UTC. As can be seen, four SNR peaks are detected. If we recall the gain pattern for beam 30, we can clearly see that these four SNR peaks may be associated to the first four gain peaks lying on $\Delta \gamma_{2}=0$, so we can imagine that the objects passed close to that line. Still, we do not know if the object entered the receiver $\mathrm{FoV}$ from the left or from the right.

For the definition of the first guess it is therefore necessary to identify some SNR peaks that are likely to be associated to specific gain peaks. The idea underlying the implemented approach consists in starting the process by considering the largest SNR peak recorded by each dominant beam. When one of these beams is illuminated, its maximum signal peak is automatically associated to the main lobe of the beam in terms of angular deviation. By assuming a linear trend in time of the angular deviation profiles $\Delta \gamma_{1}(t)$ and $\Delta \gamma_{2}(t)$ and performing a first polynomial fit, this allows us to obtain a first estimate of the trace of the object inside the receiver FoV. Once this trace is defined, we can start considering all SNR peaks, including the ones of beams with multiple comparable gain peaks, and we can associate each signal peak to a specific gain peak by identifying the lobe with minimum deviation with respect to the previously estimated trace at the epoch the SNR peak was detected. This procedure provides us with a full list including, for each recorded signal, the corresponding time epoch and angular deviations of the associated gain peaks. Finally, a new polynomial fit allows us to obtain a refined first guess for the object trace.

The described approach allows us to maximize the information extracted by the beam illumination sequence simply by looking at the recorded SNR peaks and exploiting the gain pattern. This procedure does not require any calibration of the sensor, and any other measurement apart from the SNR. If slant range measurements are also available, then a step further can be performed. Starting from the estimated object trace, and assuming a polynomial trend of the angular deviations with time, by knowing the slant range measurements at each observation instant it is possible to perform a least-squares fit aimed at minimizing the residuals with respect to the whole SNR profile of all beams. This second step, referred to as S2, allows us to increase the accuracy of the estimate and concludes the trace definition phase.

Figure 9 shows the results of the trace definition phase for object NORAD ID 41765, April 20, 2018. The real trace of the object is shown in red. On the left, in black, the estimated trace considering the largest SNR peaks of the dominant beams only 
69th International Astronautical Congress (IAC), Bremen, Germany, 1-5 October 2018. Copyright (C) 2018 by Politecnico di Milano. Published by the IAF, with permission and released to the IAF to publish in all forms.

Table 1: BIRALES OD performance for both cases of known and unknown objects.

\begin{tabular}{lllllll}
\hline Object & Noise & \#objects & $\bar{\varepsilon}_{p}(\mathrm{~km})$ & $\bar{\varepsilon}_{v}(\mathrm{~km} / \mathrm{s})$ & $\bar{\sigma}_{p}(\mathrm{~km})$ & $\bar{\sigma}_{v}(\mathrm{~km} / \mathrm{s})$ \\
\hline Known & - & 44 & $5.34 \mathrm{e}-4$ & $1.30 \mathrm{e}-4$ & $7.80 \mathrm{e}-5$ & $7.00 \mathrm{e}-6$ \\
Known & DS and SR & 44 & $7.37 \mathrm{e}-3$ & $2.45 \mathrm{e}-3$ & $6.24 \mathrm{e}-2$ & $7.45 \mathrm{e}-3$ \\
Known & DS, SR and SNR & 43 & $6.52 \mathrm{e}-2$ & $1.24 \mathrm{e}-2$ & $7.14 \mathrm{e}-2$ & $8.68 \mathrm{e}-3$ \\
\hline Unknown & - & 29 & $1.29 \mathrm{e}-3$ & $2.07 \mathrm{e}-4$ & $4.84 \mathrm{e}-4$ & $3.50 \mathrm{e}-5$ \\
Unknown & DS ans SR & 30 & $7.87 \mathrm{e}-3$ & $2.91 \mathrm{e}-3$ & $6.78 \mathrm{e}-2$ & $5.38 \mathrm{e}-3$ \\
Unknown & DS, SR and SNR & 27 & $5.39 \mathrm{e}-2$ & $1.32 \mathrm{e}-2$ & $7.47 \mathrm{e}-2$ & $4.38 \mathrm{e}-3$ \\
\hline
\end{tabular}

(beams 6,7, 15 and 24). The final result of step S1 is shown in blue: as can be seen, by considering all SNR peaks, the accuracy of the estimate significantly increases. Finally, on the right, the estimated trace obtained with step S2, considering a linear (green) and quadratic (cyan) trend in time of the angular deviations: the availability of the slant range allows us to obtain a further improvement in the definition of the trace, getting to an estimate almost overlapping the real one.

The described algorithm is valid in both cases of known object (i.e. object whose TLE is available) and unknown object. In the case of known object, the availability of the TLE gives us an a priori estimate of the possible path in the receiver FoV. This estimate can be exploited to ensure convergence of step $\mathrm{S} 2$ in all those situations in which the passage of the object is critical. These situations occur every time symmetry conditions occur. Symmetry conditions appear when a single column of gain peaks is illuminated. In such cases, the output of step S1 is a straight line connecting these lobes. If this is the case, the initial guess is exactly halfway between the real solution and a symmetric one which gives the same SNR profile, but a wrong trace in the FoV. If the object is known, convergence can be granted by deviating the initial guess of step S1 in the direction of the trace predicted by the TLE. The situation becomes more critical if the object is unknown. In this case, two possible solutions exist. The algorithm, therefore, cannot distinguish the right one, and needs to store both.

\subsection{Object state estimation}

The output of the trace definition phase provides a value of $\Delta \gamma_{1}$ and $\Delta \gamma_{2}$ for each observation instant. These two estimates, along with the Doppler shift and slant range measurements, are used to estimate the state and covariance of the object at the epoch of the first observation. The estimation is done with a Levenberg-Marquardt least-squares batch algorithm and requires an accurate model for the object dynamics. The considered highfidelity propagator, called AIDA (Accurate Integrator for Debris Analysis), includes the gravitational model EGM2008 up to order 10, the atmospheric
Table 2: RFT and Medicina receiver locations.

\begin{tabular}{lllll}
\hline & Lat & Lon & Alt & $\mathrm{P}$ \\
\hline TX & $39^{\circ} 36^{\prime} 18^{\prime \prime} \mathrm{N}$ & $9^{\circ} 26{ }^{\prime} 23^{\prime \prime} \mathrm{E}$ & $550 \mathrm{~m}$ & $10 \mathrm{~kW}$ \\
$\mathrm{RX}$ & $44^{\circ} 31^{\prime} 14^{\prime \prime} \mathrm{N}$ & $1^{\circ} 38^{\prime} 59^{\prime \prime} \mathrm{E}$ & $28 \mathrm{~m}$ & - \\
\hline
\end{tabular}

drag with the atmosphere model NRLMSISE-00, third body perturbations, and solar radiation pressure with a dual-cone model for Earth shadow for objects whose geometrical parameters are known. For unknown objects, only gravitational and thirdbody effects are considered.

\section{Results}

The analysis of the performance of BIRALES sensor is presented in this section. The first part is dedicated to the results of numerical simulation, whereas the second part shows the results obtained during the observation campaign of satellite Tiangong-1 re-entry.

\subsection{Numerical simulations}

In order to assess the performance of BIRALES sensor we performed a numerical simulation using a dedicated sensor simulator. Starting from a catalogue of available objects, the simulator computes, for each object, the predicted passage epoch and simulates the behaviour of BIRALES sensor generating the measurements in terms of slant range, Doppler shift and SNR. Further details about the behaviour of the simulator are given in 7 . We considered an observation window of two days, covering the range 5-6 September 2018, with 2490 objects with at least one passage, and for all these objects we analysed the first passage only. The characteristics of the sensor in terms location (latitude, longitude and altitude) and power are shown in Table 2 .

We performed the analysis considering both cases of known and unknown objects, assuming the following levels of measurement noise: $3 \mathrm{~m}$ of standard deviation for the slant range, $9 \mathrm{~Hz}$ of discretization for the Doppler shift, and a white Gaussian noise for the SNR, assuming a ratio of $30 \mathrm{~dB}$ 

all forms.

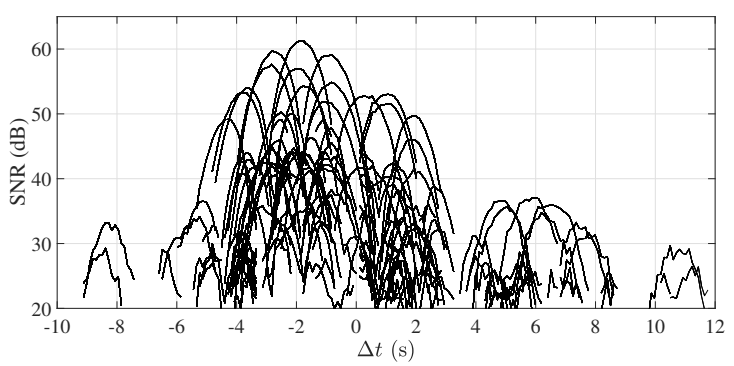

(a)

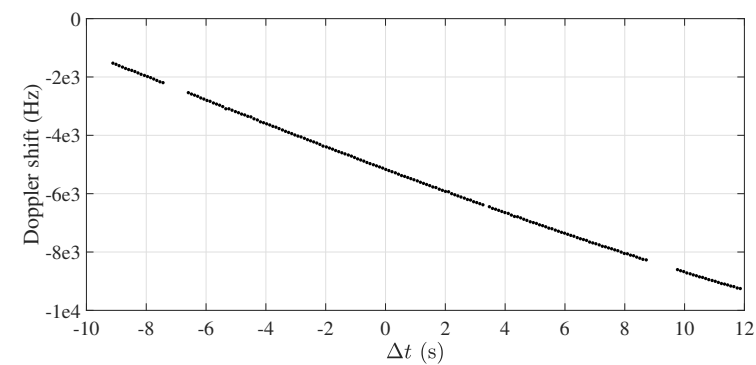

(b)

Fig. 10: SNR (a) and Doppler shift (b) measurement profiles obtained during Tiangong-1 passage on March 29, 2018 as a function of time delay $\Delta t$ (s) with respect to the TLE-predicted passage epoch.

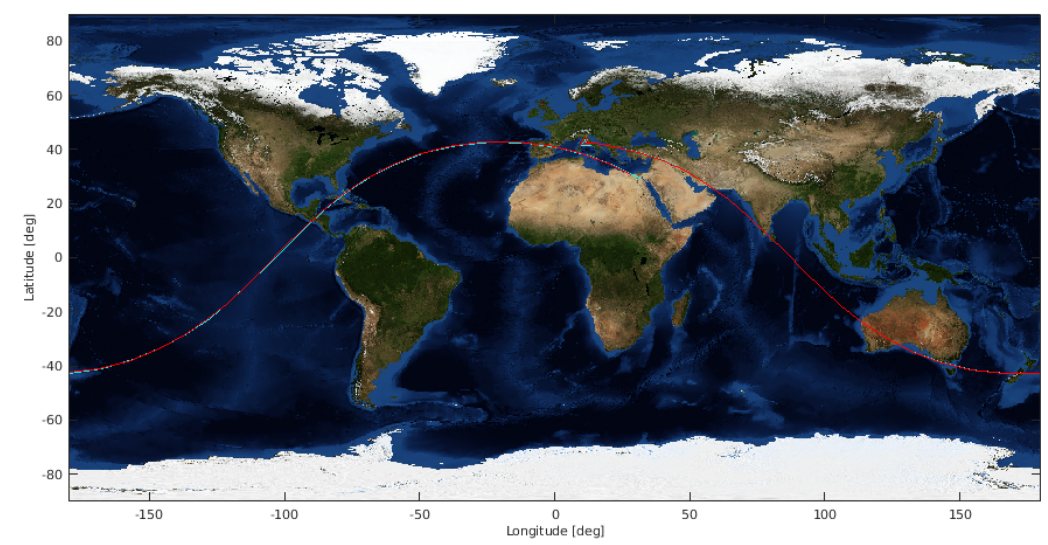

Fig. 11: Tiangong-1 trace comparison between OD (cyan) and TLE (red) estimates for March 29.

between the nominal signal and the added white noise. The results are shown in Table 1. The performance is expressed in terms of mean position and velocity errors $\bar{\varepsilon}_{p}$ and $\bar{\varepsilon}_{v}$ and mean standard deviations $\bar{\sigma}_{p}$ and $\bar{\sigma}_{v}$.

By analysing the number of observable objects, it is quite evident that this value is quite low. This is in part due to our decision of selecting the first passage only, in part is due to the peculiarity of the sensor of detecting meridian passage only. Let us now analyse the performance and consider the results for the case of known objects: as can be seen, if we do not add any kind of additional measurement noise, the accuracy granted is very high, leading to a mean position error that is less than $1 \mathrm{~m}$. If measurement noise is considered, the performance decreases, with an average decrease in accuracy of around two orders of magnitude in both position and velocity. Still, the average position error is lower than $100 \mathrm{~m}$, whereas the average velocity error is slightly larger than $10 \mathrm{~m} / \mathrm{s}$.

Let us now consider what happens in case of unknown object. As can be seen, in this case the
Table 3: BIRALES pointing directions for March $29,2018$.

\begin{tabular}{|c|c|c|c|c|}
\hline $\begin{array}{l}\text { Epoch } \\
\text { (UTC) }\end{array}$ & $\begin{array}{c}\operatorname{Az} z_{R X} \\
\left({ }^{\circ}\right) \\
\end{array}$ & $\begin{array}{c}\mathrm{El}_{\mathrm{RX}} \\
\left({ }^{\circ}\right)\end{array}$ & $\begin{array}{c}\text { AzTX }^{\circ} \\
\left({ }^{\circ}\right) \\
\end{array}$ & $\begin{array}{c}\mathrm{El}_{\mathrm{T}} \\
\left({ }^{\circ}\right)\end{array}$ \\
\hline $07: 56: 03.27$ & 180.00 & 40.83 & 28.80 & 25.61 \\
\hline
\end{tabular}

Table 4: Keplerian parameters comparison (March 29, 2018 07:55:54.35 UTC).

\begin{tabular}{lllll}
\hline & $a(\mathrm{~km})$ & $\mathrm{e}$ & $i\left(^{\circ}\right)$ & $\Omega\left(^{\circ}\right)$ \\
\hline TLE & 6567.96 & 0.0012 & 42.66 & 218.04 \\
OD & 6558.75 & 0.0028 & 42.65 & 217.96 \\
\hline
\end{tabular}

number of objects for which we could univocally provide an OD estimate decreases with respect to the case of known object, whereas the average accuracy in both position and velocity is more or less the same. This result is expected, as when the object is unknown, the trace definition algorithm is prone to symmetry problems. In this case, the only way consists in considering both possible solutions. 

all forms.

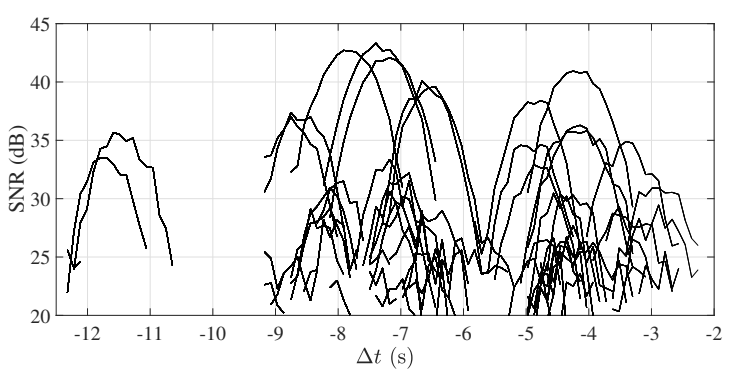

(a)

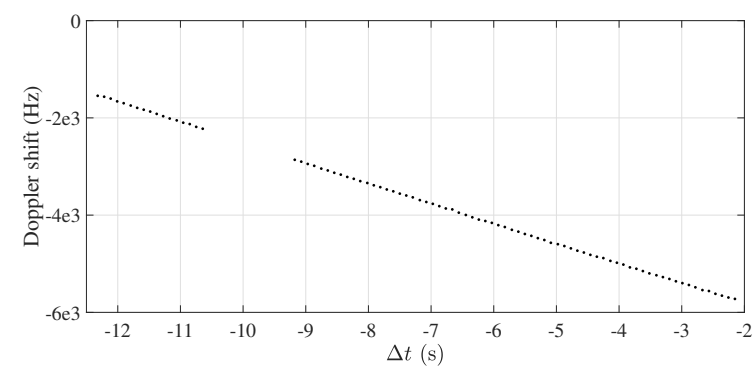

(b)

Fig. 12: SNR (a) and Doppler shift (b) measurement profiles obtained during Tiangong-1 passage on March 31, 2018 as a function of time delay $\Delta t(\mathrm{~s})$ with respect to the TLE-predicted passage epoch.

\subsection{Tiangong-1 re-entry observation campaign}

In this second part of the section, we show the results obtained during the observation campaign of Tiangong-1 re-entry. The Chinese satellite completed its re-entry on April 2, 2018 at 00:16 UTC. During the final week, BIRALES sensor was able to observe the space station several times. In the following paragraphs, we show the results obtained with the observations performed on March 29 and 31. In both observations the sensor provided only Doppler shift and SNR measurements.

Table 3 shows the sensor pointing directions for the first considered passage in terms of azimuth $(\mathrm{Az})$ and elevation $(\mathrm{El})$ and the meridian passage epoch predicted on the basis of the latest TLE available, whereas Fig. 10 shows the SNR and Doppler shift profiles as a function of the time delay with respect to the predicted meridian passage epoch. As can be seen from the SNR maximum peak, the meridian passage occurred around $2 \mathrm{~s}$ in advance with respect to what was expected. Given the unavailability of the slant range, the trace of the object was estimated just relying on step S1, only considering the SNR peaks. The obtained angular deviation estimates were coupled with the measured Doppler shift, and a least-squares fit was used to obtain an estimate of the state of the object at the epoch of the first observation. Figure 11 shows the comparison between the estimated trace (cyan) and the one provided by the latest TLE for one satellite orbit. A comparison between the estimated orbital parameters (semi-major axis $a$, eccentricity $e$, orbital plane inclination $i$ and right ascension of the ascending node $\Omega$ ) is shown in Table 4 . As can be seen, the most significant differences can be detected in the orbital eccentricity and the semimajor axis, whereas both inclination and right ascension of the ascending node are very similar.

A second passage was detected on March 31, 2018. Table 5 shows the TLE-predicted pointing directions and the expected meridian passage epoch. In this case, the difference between the TLE
Table 5: BIRALES pointing directions for March 31,2018 .

\begin{tabular}{|c|c|c|c|c|}
\hline $\begin{array}{l}\begin{array}{l}\text { Epoch } \\
\text { (UTC) }\end{array} \\
\end{array}$ & $\begin{array}{c}\begin{array}{c}\text { AzRX } \\
\left({ }^{\circ}\right)\end{array} \\
\end{array}$ & $\begin{array}{c}\mathrm{El}_{\mathrm{RX}} \\
\left({ }^{\circ}\right)\end{array}$ & $\begin{array}{c}z_{\text {TXX }} \\
\left({ }^{\circ}\right)\end{array}$ & $\begin{array}{c}\mathrm{El}_{\mathrm{T}} \\
\left({ }^{\circ}\right)\end{array}$ \\
\hline $06: 54: 11.61$ & 180.00 & 38.78 & 28.54 & 23.40 \\
\hline
\end{tabular}

Table 6: Keplerian parameters comparison (March 31, 2018 06:53:59.29 UTC).

\begin{tabular}{lllll}
\hline & $a(\mathrm{~km})$ & $\mathrm{e}$ & $i\left(^{\circ}\right)$ & $\Omega\left(^{\circ}\right)$ \\
\hline TLE & 6551.48 & 0.0009 & 42.67 & 205.05 \\
OD & 6538.00 & 0.0023 & 42.67 & 205.27 \\
\hline
\end{tabular}

prediction and the real passage was more significant (around $7 \mathrm{~s}$ ), as can be seen by looking at the recorded SNR and DS profiles (Fig. 12). This yielded a more evident difference between the orbital parameters provided by the TLE and the estimated ones, as shown in Table 6 .

Starting from the available state estimates, an estimation for the re-entry epoch was obtained by propagating them forward in time. Figure 13 shows the evolution in time of the predicted re-entry window, provided by the European Space Agency. On the same plot, the re-entry estimates obtained with the observations of March 29 and 31 are shown. As can be seen, while the first estimate is out of the predicted window, the estimate obtained with the observation of March 31 is well included in the predicted re-entry window. This result is quite important, since it shows that BIRALES sensor can provide sufficiently accurate results even with only Doppler shift and SNR measurements starting from the estimates of a single object passage. Considering the current availability of additional slant range measurement, we are confident that the good performance shown by the sensor will be further improved in future observation campaigns. In any case, the obtained results show that BIRALES sen- 
69th International Astronautical Congress (IAC), Bremen, Germany, 1-5 October 2018. Copyright (C) 2018 by Politecnico di Milano. Published by the IAF, with permission and released to the IAF to publish in all forms.

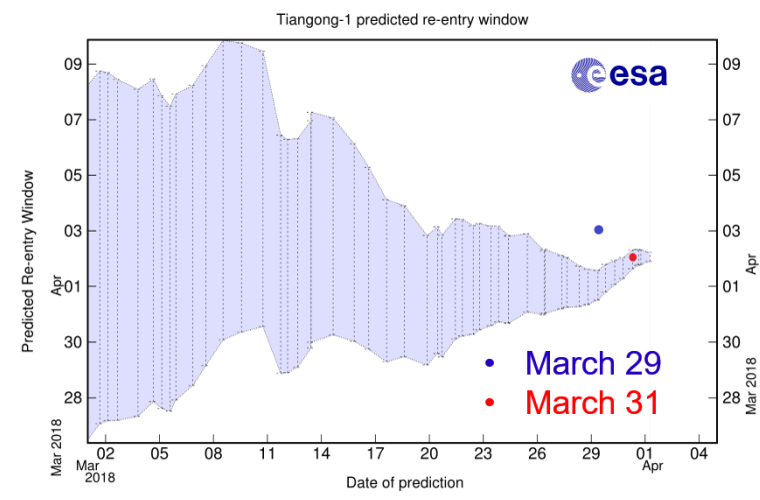

Fig. 13: Estimated re-entry window for satellite Tiangong-1.

sor could play an important role in the framework of a national and European sensor network.

\section{Conclusions}

The performance of BIRALES sensor in terms of achievable accuracy of the orbital estimation process on a catalogue of resident space objects has been assessed through numerical simulations. The sensor can estimate the orbital states with reasonable accuracy with just a single pass for both cases of known and unknown objects. The latter represents the most critical one, as sometimes symmetry problems lead to two possible solutions. The results obtained during satellite Tiangong-1 re-entry observation campaign have been shown, and the good agreement between predicted and estimated object state was highlighted. Future developments include the testing of the sensor including slant range measurements, and the possible coupling with other Italian and European radar sensors.

\section{Acknowledgements}

The authors acknowledge the support of the Italian Space Agency and the Italian National Institute of Astrophysics through the grant agreement n. 2015-028-R.O. (Space Debris - IADC activities support and SST pre-operative validation). The research activities and operations described in this paper were performed within the European Commission Framework Programme H2020 and Copernicus "SST - Space Surveillance and Tracking" contracts No. 785257-2-3SST2016 and No. 237/G/GRO/COPE/16/8935-1SST2016. The Radio Frequency Transmitter is a facility of the Italian Air Force, located at Italian Joint Test Range of Salto di Quirra in Sardinia. The Northern Cross Radio Telescope is a facility of the University of Bologna operated under agreement by the IRAINAF (Radio Astronomy Institute - National In- stitute of Astrophysics). The authors acknowledge Daniele Antonio Santeramo from Politecnico di Milano for his contribution to the graphical elaboration of Tiangong-1 re-entry OD results. M. Losacco gratefully acknowledges the Italian Space Agency and the Space Generation Advisory Council for the 2018 ASI/SGAC grant.

\section{References}

[1] T. Flohrer, H. Krag, S. Lemmens, B. Bastida Virgili, K. Merz, and H. Klinkrad, "A Statistical Look at ESA's Conjunction Event Predictions," 6th European Conference on Space Debris, Darmstadt, Germany, Apr. 2013.

[2] C. Pardini and L. Anselmo, "Re-entry predictions of three massive uncontrolled spacecraft," 23rd International Symposium on Space Flight Dynamics, Pasadena, California, Oct. 2012.

[3] E. Parliament and Council, "Decision No 541/2014/EU of the European Parliament and of the Council of 16 April 2014 establishing a Framework for Space Surveillance and Tracking Support," http://data.europa.eu/eli/dec/ 2014/541(1)/oj, Apr. 2014.

[4] ESA, "ESA's space situational awareness," http://www.esa.int/Our_Activities/ Operations/Space_Situational_Awareness/ About_SSA, Mar. 2017. (accessed on March 29, 2017).

[5] A. Morselli, P. Di Lizia, G. Bianchi, C. Bortolotti, S. Montebugnoli, G. Naldi, F. Perini, G. Pupillo, M. Roma, M. Schiaffino, A. Mattana, E. Salerno, A. Magro, K. Z. Adami, R. Armellin, A. Lazzareschi Sergiusti, W. Villadei, F. Dolce, M. Reali, and J. Paoli, "A new high sensitivity radar sensor for space debris detection and accurate orbit determination," Metrology for Aerospace (MetroAeroSpace), Rome, Italy, June 2015.

[6] S. Montebugnoli, G. Bianchi, J. Monari, G. Naldi, F. Perini, and M. Schiaffino, "BEST: Basic Element for SKA Training.," Wide Field Astronomy Technology for the Square Kilometre Array, Chateau de Limelette, Belgium, Nov. 2009.

[7] M. Losacco, P. Di Lizia, M. Massari, A. Mattana, F. Perini, M. Schiaffino, C. Bortolotti, M. Roma, G. Naldi, G. Pupillo, G. Bianchi, D. Cutajar, A. Magro, C. Portelli, M. Reali, and W. Villadei, "Orbit determination of resident space objects with the multibeam radar sensor BIRALES," AIAA SciTech Forum, Kissimmeee, Florida, Jan. 2018. 